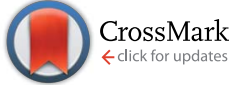

Cite this: RSC Adv., 2015, 5, 79600

\title{
Preparation and characterisation of transparent and flexible PEDOT:PSS/PANI electrodes by ink-jet printing and electropolymerisation
}

\author{
Annalisa Vacca, ${ }^{\text {*a }}$ Michele Mascia, ${ }^{a}$ Simone Rizzardini, ${ }^{a}$ Simona Corgiolu, ${ }^{a}$ \\ Simonetta Palmas, ${ }^{a}$ Monia Demelas, ${ }^{b}$ Annalisa Bonfiglio ${ }^{\mathrm{b}}$ and Pier Carlo Riccic
}

Novel flexible PEDOT:PSS/PANI bilayered thin-film electrodes were successfully prepared by ink-jet printing of PEDOT:PSS on a PEN substrate and subsequent electrochemical polymerisation of PANI. Scanning electron microscopy (SEM), cyclic voltammetry (CV) and electrochemical impedance spectroscopy (EIS) were used to systematically investigate the structure, morphology and electrochemical behaviour of the electrodes. The PEDOT:PSS/PANI bilayer nanofilms have been tested as $\mathrm{pH}$ sensors. As a comparison, either PEDOT:PSS or PANI electrodes have been tested for $\mathrm{pH}$ measurements. The results show that the sensitivity of the PEDOT:PSS/PANI bilayer film is higher than that of PEDOT:PSS and PANI electrodes, indicating a synergistic effect of the two polymers. A reversible near-Nerstian behaviour of the open circuit voltage with $\mathrm{pH}$ was found, with a slope of $58.9 \mathrm{mV} \mathrm{pH}^{-1}$ unit in a wide range of $\mathrm{pH}$ values from 2 to 14.

Received 31st July 2015

Accepted 15th September 2015

DOI: $10.1039 / \mathrm{c} 5 \mathrm{ra15295j}$

www.rsc.org/advances

the PEDOT:PSS particles dispersed in water; however, the pris-

\section{Introduction}

Conducting polymers have attracted much attention since their discovery in the late 1970's. Thanks to their outstanding characteristics such as light-weight and high flexibility, the use of polymer as active materials in electronic devices may allow replacement of the traditional metal-based devices and obtain fully-plastic electronic devices. ${ }^{1}$ One of the technical challenges is due to the very low conductivity of some solution-processible organic compounds, which can lower the device performance.

Among others, poly(3,4-ethylenedioxythiophene) (PEDOT) presents very interesting properties including high electrical conductivity, environmental stability, and high transparency, ${ }^{1,2}$ so that it has been proposed for different electronics applications, such as organic photovoltaic devices (OPVs) ${ }^{3}$ and organic light emitting diodes (OLEDs). ${ }^{4}$ Moreover, PEDOT could be patterned by an ink-jet printing technique, which is a low-cost, simple, easy method to fabricate flexible devices. ${ }^{5}$ However, PEDOT is not soluble in water, so that aqueous inks are usually prepared using poly(styrenesulfonic acid) (PSS): the formation of the polyelectrolyte complex of PEDOT:PSS enhances the dispersibility of particles in water. ${ }^{6}$ The conductivity of the film depends on the ratio of PEDOT to PSS as well as on the size of

\footnotetext{
${ }^{a}$ Dipartimento di Ingegneria Meccanica Chimica e dei Materiali, Università degli Studi di Cagliari, Via Marengo 2, 09123 Cagliari, Italy. E-mail: annalisa.vacca@dimcm. unica.it; Tel: +390706755059

${ }^{b}$ Dipartimento di Ingegneria Elettrica ed Elettronica, Università degli Studi di Cagliari, Via Marengo 3, 09123 Cagliari, Italy

'Dipartimento di Fisica, Università degli Studi di Cagliari, Complesso Universitario di Monserrato, S.P. Monserrato-Sestu Km 0.700, 09042 Monserrato (CA), Italy
} tine films present a very low conductivity $\left(<1 \mathrm{~S} \mathrm{~cm}^{-1}\right)$ to be used as electrode material. ${ }^{7}$ Several authors attribute the low conductivity of the PEDOT:PSS films to the formation of coil structures, in which PEDOT is surrounded by PSS, in a discontinuous granular system with low interconnections between the particles. ${ }^{1,6,8,9}$ To overcome this drawback, different compounds have been added during the preparation: noticeable increasing in conductivity (up to 2 or 3 orders of magnitude) has been obtained by using surfactants, ${ }^{10}$ graphene oxide $^{6-11}$ or polar solvents ${ }^{12}$ which can change the pristine structure. For example, by using ethylene glycol (EG) the dedoping process can partially remove the amorphous and insulating PSS phase improving the conductivity up to $467 \mathrm{~S} \mathrm{~cm}^{-1} \cdot{ }^{13}$

Another strategy is the combination of PEDOT:PSS with other conducting polymers, which can allow obtaining hybrid organic systems with tuneable properties: in particular polyaniline (PANI) presents very attractive characteristics including high conductivity and high active surface due to its porous structure. PANI is generally synthesized by chemical or electrochemical oxidative polymerisation of the monomer in acidic aqueous medium: however, the electrochemical polymerisation allows to a better control of the synthesis parameters. Many characteristics of the PANI depend on the redox equilibria of various reduced and oxidized forms, which take place in accordance with redox potential and $\mathrm{pH}$ of the media. ${ }^{14,15} \mathrm{In}$ particular, the half-oxidized form of PANI originates the deprotonated emeraldine base and the protonated emeraldine salt which is the most electrically conductive form. PANI is then usually active at acidic $\mathrm{pH}$ while its activity decreases at neutral and alkaline $\mathrm{pH}$. PANI can be easily combined with anionic 
polymers and polyelectrolytes to form layer-by-layer constructions: the formation of multi-layered complexes of PANI with negatively charged components can enhance the redox activity of the polymer at higher $\mathrm{pH}^{16}$

Combinations of different conducting polymers can allow obtaining composites which present novel proprieties and unique structures. ${ }^{17}$ Composites based on PEDOT:PSS and PANI have been prepared following different strategies allowing to obtain powders or multilayer composites. Zhang and coworkers prepared PEDOT/PANI bilayer nanofibers by subsequent oxidative polymerization of the parent monomers in bulk solution: the obtained nanofibers were used as electrode after a drop-cast onto a glassy carbon. ${ }^{18}$ Bilayered nanofilms of PEDOT/ PANI have been synthetized by Shi and coworkers by electrochemical polymerization of PANI in a mixed alcohol solution on PEDOT:PSS nanofilm electrode prepared by spin coating of PEDOT:PSS $/ 5 \%$ DMSO on glass. ${ }^{19}$ Similarly, the same group prepared bilayered PEDOT/PANI electrodes starting form electrodes based on single-walled carbon nanotubes and PEDOT:PSS spin coated on glass. ${ }^{20}$

In this work, flexible and transparent fully organic hybrid electrodes have been prepared by ink-jet printing of PEDOT:PSS on polyethylene naphtalate (PEN) substrates followed by electrochemical polymerisation of PANI. This approach conjugates the ink-jet printing technique, which is a low-cost and easily scalable method to obtain flexible devices with the electrochemical polymerization which allows a good control and adhesion of the PANI layer on PEDOT:PSS.

\section{Experimental section}

The PEDOT:PSS electrodes are fabricated on a poly(ethylene 2,6naphthalate) (PEN) substrate, $125 \mu \mathrm{m}$ thick. Substrates are cleaned by subsequent $10 \mathrm{~min}$ of ultrasonic bath in water and in acetone; they are washed in deionized water, dried under nitrogen flow and then activated by means of a plasma treatment $(10 \mathrm{~s}$ at $100 \mathrm{~W})$. The PEDOT:PSS layer is realized by ink-jet printing using the Fujifilm Dimatix Material Printer (DMP) 2800 . The ink is prepared by sonicating for 20 minute a solution made of PEDOT:PSS (pH 500 provided from H. C. Starck). Three layers are printed by using three nozzles of a DMC-11601 cartridge (each nozzle generates $1 \mathrm{pL}$ drop of ink) with a drop spacing of $15 \mu \mathrm{m}$. During printing, substrates are kept at $45^{\circ} \mathrm{C}$ to facilitate the solvent evaporation. After printing, samples are annealed in an oven at $130{ }^{\circ} \mathrm{C}$ for $15 \mathrm{~min}$. After annealing, ethylene glycol is deposited by spin coating and dried in an oven at $130{ }^{\circ} \mathrm{C}$ for $15 \mathrm{~min}$. These electrodes are denoted as PEDOT:PSS (see Fig. 1).

The electrochemical experiments were performed at room temperature using an AUTOLAB PGSTAT302N (Metrohm, Switzerland) potentiostat/galvanostat equipped with a frequency response analyser controlled with the NOVA software. A conventional three-electrode cell $(V=10 \mathrm{~mL})$ was used in which Saturated Calomel Electrode (SCE) was the reference and Pt wire was used as counter electrode. Polyaniline was electropolymerized on PEDOT:PSS electrodes from aqueous solutions of aniline $(0.1 \mathrm{M})$ and $\mathrm{HNO}_{3}(1 \mathrm{M})$. Electropolymerisation was

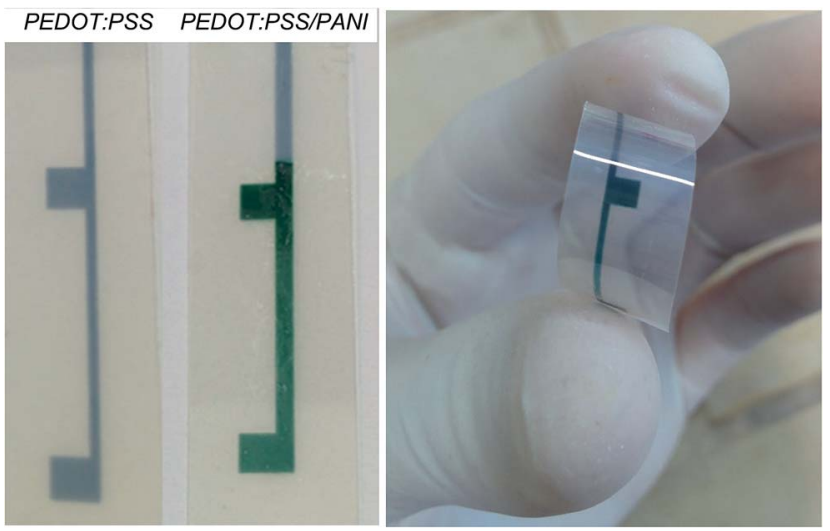

Fig. 1 Pictures of the ink-jet printed PEDOT:PSS and PEDOT:PSS/ PANI electrodes.

performed by cyclic voltammetries from 0 to $0.95 \mathrm{~V}$ with a scan rate of $100 \mathrm{mV} \mathrm{s}^{-1}$. Ten cycles were applied to obtain PANI films. The electrodes obtained by electropolymerisation on PEDOT: PSS are denoted as PEDOT:PSS/PANI (see Fig. 1).

The electrodes were characterized by cyclic voltammetries (CVs), electrochemical impedance spectroscopies (EIS) and open circuit potential (OCP) measurements in solution with different $\mathrm{pH}$. EIS were performed in solution with different $\mathrm{pH}$ by varying the frequency from $30 \mathrm{kHz}$ down to $0.1 \mathrm{~Hz}$ at open circuit potential with an excitation amplitude of $10 \mathrm{mV}$. The impedance spectra were then fitted to an equivalent electrical circuit by using the software ZSimpWin 2.0 (EChem software). Scanning electron microscopy (NOVA 600, Nanolab dual beam) was used to characterize the structures of PEDOT:PSS and PEDOT:PSS/PANI electrodes. Focused ion beam (FIB) was also used to very precise cross sections of samples for subsequent imaging via SEM.

Raman measurements were performed in air at room temperature with a compact spectrometer BWTEK i-Raman Ex integrated system, excitation wavelength at $1064 \mathrm{~nm}$ (provided by a Nd:YAG laser), in back scattering geometry. The spectra were collected with very low power beam $(<5 \mathrm{~mW})$ focalized in $1 \mathrm{~mm}^{2}$ through a microscope objective Olympus $\times 20$ to avoid any heating or photodegradation effect. The threshold to observe the photodegradation of the sample (pure polyaniline) was observed for beam power higher than $15 \mathrm{~mW}$.

\section{Results and discussion}

Fig. 2 shows the cyclic voltammogram of aniline swept at $100 \mathrm{mV} \mathrm{s}^{-1}$ during 10 scans in aqueous solution containing $0.1 \mathrm{M}$ aniline and $1.0 \mathrm{M} \mathrm{HNO}_{3}$ at PEDOT:PSS working electrode. The electropolymerisation process of PANI on conducting electrodes (i.e. gold, platinum) usually exhibits well-defined redox peaks corresponding to a series of redox transitions, which are related to the polymerisation processes. ${ }^{21}$ In the present case, two oxidation waves and one reduction wave can be identified (see inset of Fig. 1), although with difficult due to the low conductivity of the PEDOT electrode. Anyway, both 


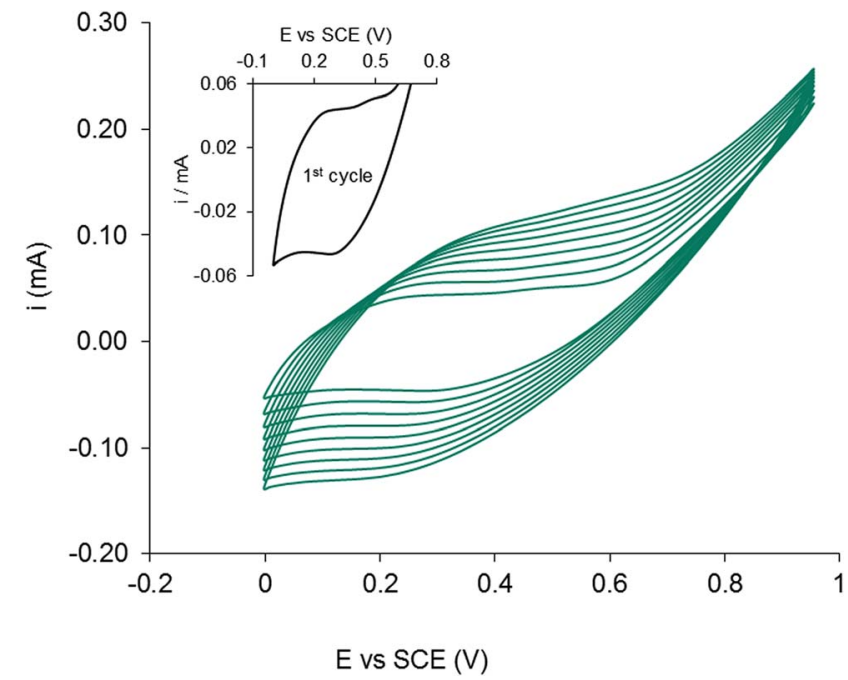

Fig. 2 Cyclic voltammograms recorded during PANI electropolymerisation in $0.1 \mathrm{M}$ aniline $+1 \mathrm{M} \mathrm{HNO}_{3}$ aqueous solution on PEDOT:PSS electrodes. Inset shows the first voltammetric cycle of polymerisation.

oxidation and reduction currents progressively increase with the scans, thus evidencing the growing of a conductive film on the electrode surface. The thickness of the deposited layer of PANI can be estimated from the voltammetric charge associated to the oxidation of the monomer $\left(Q_{\mathrm{a}}\right)$ by assuming a current yield of 1 , as follows: ${ }^{22}$

$$
h=\frac{Q_{\mathrm{a}} M_{\mathrm{w}}}{z F d A}
$$

where $M_{\mathrm{w}}$ is the molecular weight of aniline, $z=0.5$ (number of electrons/aniline), $A$ is the area of the electrode, $d$ is the specific density of aniline, and $F$ is Faraday's constant. After ten cycles, the estimated thickness of the PANI coating is about $305 \mathrm{~nm}$ and a green layer is visible on the grey surface of PEDOT:PSS.

The surface morphology of the electrodes has been observed by SEM: Fig. 3 shows the micrographs of ink-jet PEDOT:PSS and PEDOT:PSS/PANI films. As it can be seen, the PEDOT:PSS layer is uniform and relatively smooth; after the electropolymerisation of PANI a porous layer with a fibrous nanometric structure is visible, which covers the under-layer of PEDOT:PSS. The FIB cross section shows that the PEDOT:PSS layer is about $930 \mathrm{~nm}$, while after the PANI electropolymerisation the overall thickness of the layer is $1300 \mathrm{~nm}$ with non-uniform profile.

Fig. 4 reports the Raman spectra collected on PEDOT:PSS/ PANI sample, and, for comparison, the spectrum of pure polyaniline and from PEDOT:PSS. As can be observed the Raman spectrum of the PEDOT:PSS/PANI film presents all the main vibrations of polyaniline: at $1170 \mathrm{~cm}^{-1}(\mathrm{C}-\mathrm{H}$ bending vibration of the quinoid/benzenoid ring), the $\mathrm{C}-\mathrm{N}^{+}$vibration at $1327 \mathrm{~cm}^{-1}$, the vibration due to delocalized polaron in the extended polymeric conformation at $1365 \mathrm{~cm}^{-1}$, the band to the $\mathrm{N}-\mathrm{H}$ vibration at about $1500 \mathrm{~cm}^{-1}$ and, finally the band at $1595 \mathrm{~cm}^{-1}$, related to the stretching vibration of $\mathrm{C}-\mathrm{C}$ in the benzenoid ring. ${ }^{23}$ Moreover, the band related to the PEDOT:PSS

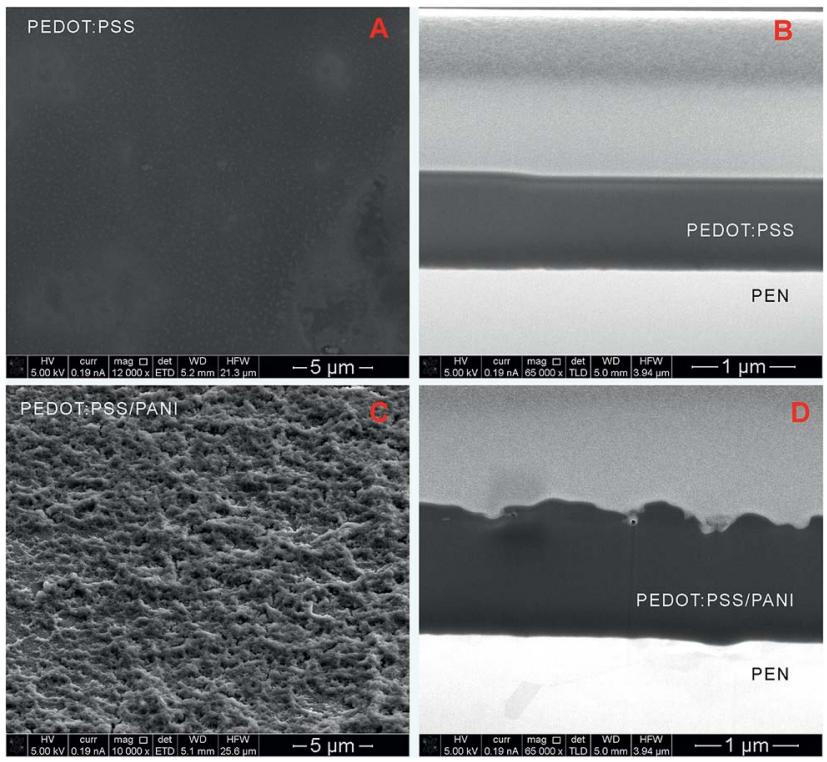

Fig. 3 SEM micrograph of surfaces ( $A$ and $C$ ) and of the focused ion beam (FIB) cross sections (B and D) of PEDOT:PSS ( $A$ and $B$ ) and PEDOT:PSS/PANI (C and D) on PEN substrate.

at about $1250 \mathrm{~cm}^{-1}$ and at $1422 \mathrm{~cm}^{-1}$ are still visible. Actually, the IR laser beam at $1064 \mathrm{~nm}$ is not totally absorbed from the polyaniline thin film, being able to reach and stimulate the organic substrate. On the other hand, the low energy laser beam avoid any luminescence effect from polyaniline.

The presence of electroactive PANI layer on the PEDOT:PSS substrate can be evidenced by cyclic voltammetries in monomer-free solutions. Fig. 5 shows the voltammograms of PEDOT:PSS and PEDOT:PSS/PANI electrodes in $1 \mathrm{M} \mathrm{HNO}_{3}$. The $\mathrm{CV}$ at PEDOT:PSS electrode shows a nearly rectangular shape, and only two redox shoulders are present at 0.6 and $0.26 \mathrm{~V}$, indicating low faradic activity and highly capacitive behaviour. ${ }^{24}$ Similar results have been already observed for PEDOT inkjet

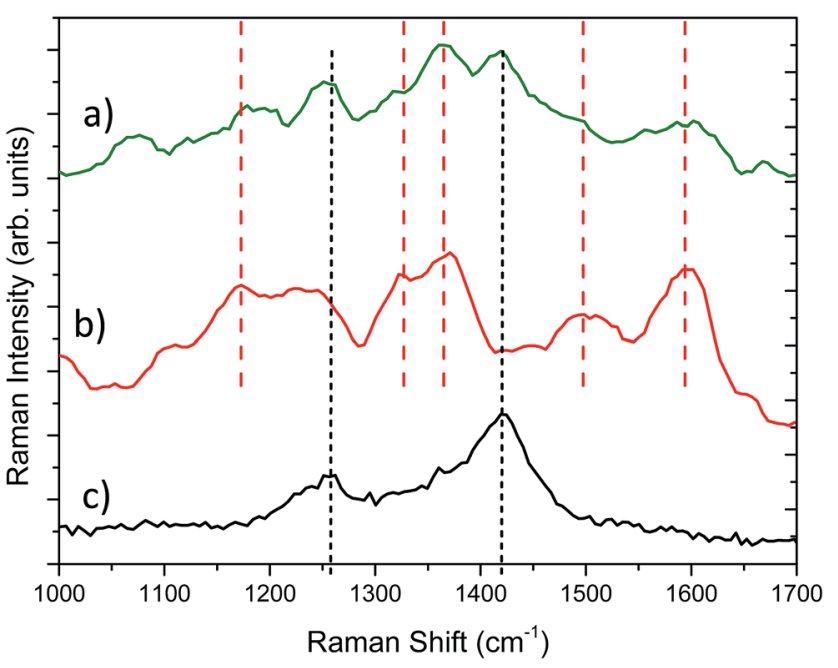

Fig. 4 Raman spectra of PEDOT:PSS/PANI (a), PANI (b) and PEDOT:PSS (c). 


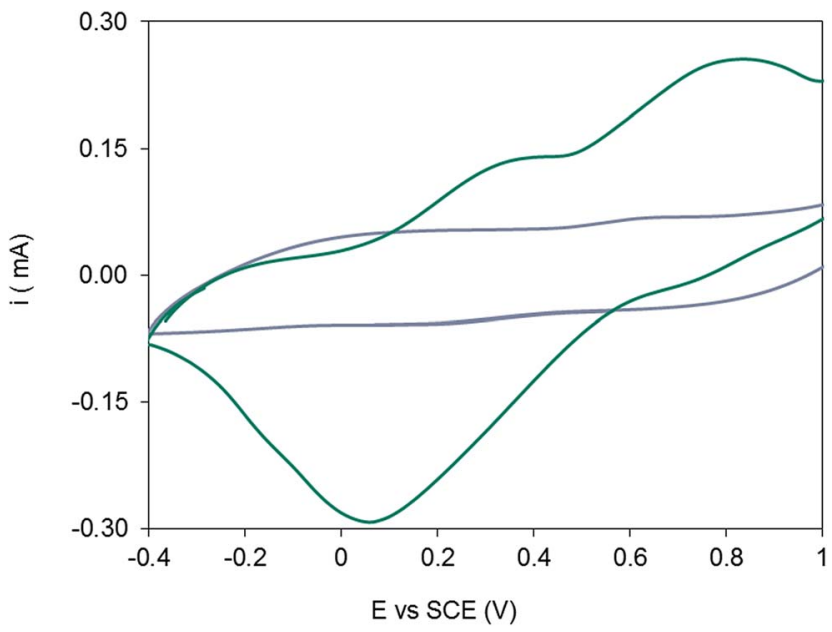

Fig. 5 Cyclic voltammetries of PEDOT:PSS (grey line) and PEDOT:PSS/PANI (green line) electrodes performed in monomer-free solutions containing $1 \mathrm{M} \mathrm{HNO}_{3}$. Scan rate $100 \mathrm{mV} \mathrm{s}^{-1}$.

printed on flexible material without metal current collector, which are similar to those used in the present work. ${ }^{25}$ In the CV of PANI modified electrode, the typical peaks related to doping and undoping of protons and anions in the polymeric film, as well as to the transformation between different forms of PANI, are well visible. The oxidation peak around $+250 \mathrm{mV}$ is attributed to the conversion from leucoemeraldine into emeraldine form, while the second peak appearing around $+800 \mathrm{mV}$ is due to emeraldine/pernigraniline transformation. ${ }^{26-28}$ Moreover, comparing the CVs of PEDOT:PSS and PEDOT:PSS/PANI it can be observed that the capacitance in presence of PANI is considerably larger than in case of PEDOT:PSS, thus indicating that PEDOT:PSS/PANI has higher electrochemical active area.

The electrochemical proprieties of the polymeric electrodes have been investigated by electrochemical impedance spectroscopy in aqueous solutions at different pH. Fig. 6 shows the Nyquist plots of PEDOT:PSS and PEDOT:PSS/PANI, recorded in acidic solution at the OCP: as can be seen for PEDOT the impedance plot presents a $90^{\circ}$ capacitive line in the low frequencies range, while at higher frequencies two deviations can be observed (see magnification of Nyquist plot in the inset of Fig. 6).

The high-frequency intercept of the semicircle with the real axis can be used to evaluate the internal resistance, which includes the resistance of the electrolyte solution, the intrinsic resistance of the active material, and the contact resistance at the electroactive material/current collector interface. ${ }^{29,30}$ PEDOT:PSS and PEDOT:PSS/PANI show similar impedance spectra, but the second one is shifted to the left, indicating a less resistive behaviour. In order to investigate the effect of the presence of PANI over the PEDOT, some electrodes with an higher PANI load have been prepared by 20 cycles of voltammetric polymerisation (PEDOT:PSS/PANI20): the EIS spectrum of one of them is compared with the previous one in Fig. 6. As can be seen, the reduction of the resistivity due to PANI layer is emphasised in samples with higher PANI load.

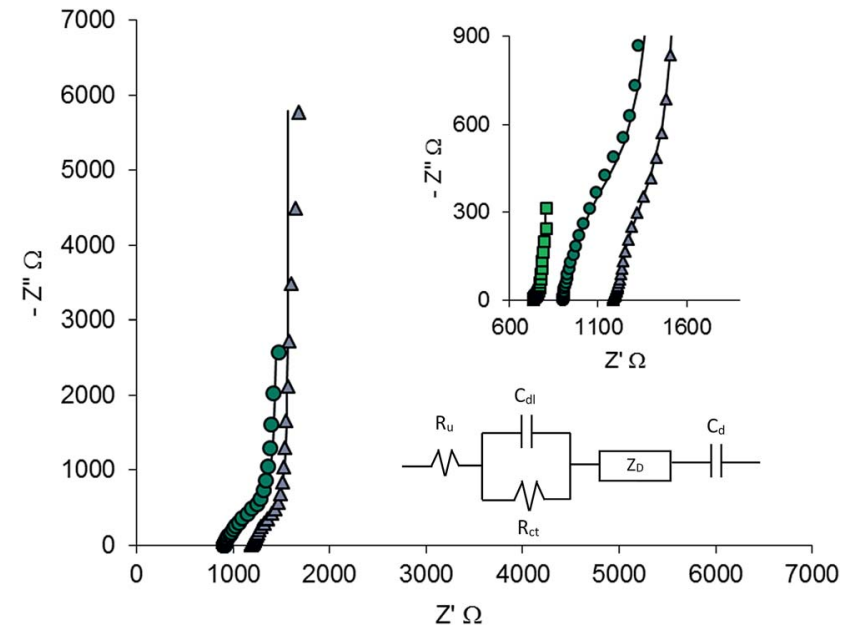

Fig. 6 Nyquist plots of PEDOT:PSS (triangles), PEDOT:PSS/PANI (circles) and PEDOT:PSS/PANI20 (squares) recorded in solution at $\mathrm{pH}=2.8$.

In order to obtain the characteristic parameters of the electrodes, the impedance spectra have been modelled with the equivalent circuit proposed by Danielsson et $a .^{31}$ for PEDOT electrodes (see inset of Fig. 6). The following elements have been inserted in the circuit: the uncompensated resistance $\left(R_{\mathrm{u}}\right)$, the charge transfer resistance $\left(R_{\mathrm{ct}}\right)$, the double layer capacitance $\left(C_{\mathrm{dl}}\right)$, the electronic contribution to the capacitance $\left(C_{\mathrm{d}}\right)$ and the finite-length Warburg element $\left(Z_{\mathrm{D}}\right)$ which represents the diffusion within a thin layer of electrolyte. $Z_{\mathrm{D}}$ includes a diffusion capacitance $\left(C_{\mathrm{D}}\right)$, a diffusional time constant $\left(\tau_{\mathrm{D}}\right)$ and a diffusion resistance $\left(R_{\mathrm{D}}=\tau_{\mathrm{D}} / C_{\mathrm{D}}\right):^{32,33}$

$$
Z_{\mathrm{D}}=\frac{\left(\tau_{\mathrm{D}} / C_{\mathrm{D}}\right) \operatorname{coth}\left(j \omega \tau_{\mathrm{D}}\right)^{1 / 2}}{\left(j \omega \tau_{\mathrm{D}}\right)^{1 / 2}}
$$

where $\omega$ is the angular frequency $(2 \pi f)$ and $j=(-1)^{1 / 2}$.

All the impedance spectra obtained for PEDOT:PSS, PEDOT:PSS/PANI and PEDOT:PSS/PANI20 have been modelled with this equivalent circuit obtaining a very good fit of the impedance spectra $\left(\chi^{2}<6 \times 10^{-5}\right)$. The fitting curves of the Nyquist plots can be seen in Fig. 6 , while Table 1 reports the values of the circuit parameters. As can be seen in Table 1 , the uncompensated resistance for PEDOT:PSS is $1180 \Omega$, indicating high intrinsic resistance of this electrode. After the electropolymerisation of PANI on PEDOT:PSS, $R_{\mathrm{u}}$ decreases: values of 900 and $733 \Omega$ are obtained for PEDOT:PSS/PANI and

Table 1 Values of the elements calculated from model of the equivalent circuit fitted to impedance spectroscopy data at $\mathrm{pH} 2.8$

\begin{tabular}{llll}
\hline & PEDOT:PSS & PEDOT:PSS/PANI & PEDOT:PSS/PANI20 \\
\hline$R_{\mathrm{u}}(\mathrm{ohm})$ & 1183 & 900 & 734.2 \\
$C_{\mathrm{dl}}(\mathrm{mF})$ & 0.33 & 0.40 & 0.66 \\
$R_{\mathrm{ct}}(\mathrm{ohm})$ & 171.8 & 66 & 13.2 \\
$C_{\mathrm{d}}(\mathrm{mF})$ & 0.31 & 0.72 & 6.37 \\
$\tau_{\mathrm{D}}(\mathrm{s})$ & 1.44 & 3.92 & 7.21 \\
$C_{\mathrm{D}}(\mathrm{mF})$ & 2.26 & 4.81 & 34.77
\end{tabular}


PEDOT:PSS/PANI20 respectively. Similarly, the charge transfer resistance decreases after the PANI deposition and the lowest values correspond to the higher PANI load. As the capacitance is concerned, the total interfacial capacitance $C_{\text {tot }}$ of the polymers can be evaluated combining in series the electronic contribution to the bulk capacitance $C_{\mathrm{d}}$ and the ionic contribution to the bulk capacitance $C_{\mathrm{D}}$ as follow: ${ }^{33,34}$

$$
C_{\mathrm{tot}}=\frac{1}{1 / C_{\mathrm{d}}+1 / C_{\mathrm{D}}}
$$

As can be seen for PEDOT:PSS $C_{\mathrm{d}} \ll C_{\mathrm{D}}$ meaning that the total interfacial capacitance is dominated by the electronic contribution: similar behaviour has been found by other authors for PEDOT:PSS electrodes. ${ }^{33,34}$ For PEDOT:PSS modified with PANI, the ionic contribution becomes more important due to the porous structure of PANI: as the PANI load increases the diffusional time constant increases indicating a slower access of the counter ions into the pores of polymer.

The electrochemical activity of the organic bilayer has been tested via measurements of $\mathrm{pH}$ vs. OCP. Fig. 7 shows the behaviour of PEDOT:PSS/PANI electrode at different $\mathrm{pH}$ : the potential equilibrium response shows a linear trend with $\mathrm{pH}$ in the range from 2 to 13.5 , with a slope of $58.9 \mathrm{mV} \mathrm{pH}^{-1}$ unit which indicates a near-Nerstian behaviour of the organic bilayer. In order to investigate the reversibility of the response to $\mathrm{pH}$, PEDOT:PSS/PANI electrode was tested with increasing and decreasing $\mathrm{pH}$. As can be seen from Fig. 6 the potentiometric responses did not show significant dependence on the direction of $\mathrm{pH}$ changes indicating a very low hysteresis. Different results were obtained for PEDOT:PSS/PANI20: the trend of OCP vs. $\mathrm{pH}$ showed two slopes of $51.1 \mathrm{mV} \mathrm{pH}^{-1}$ unit and $8.9 \mathrm{mV} \mathrm{pH}^{-1}$ unit in acidic and neutral-alkaline $\mathrm{pH}$, respectively.

Moreover, measurements of OCP vs. $\mathrm{pH}$ have been performed using electrodes of either PANI or PEDOT:PSS onto

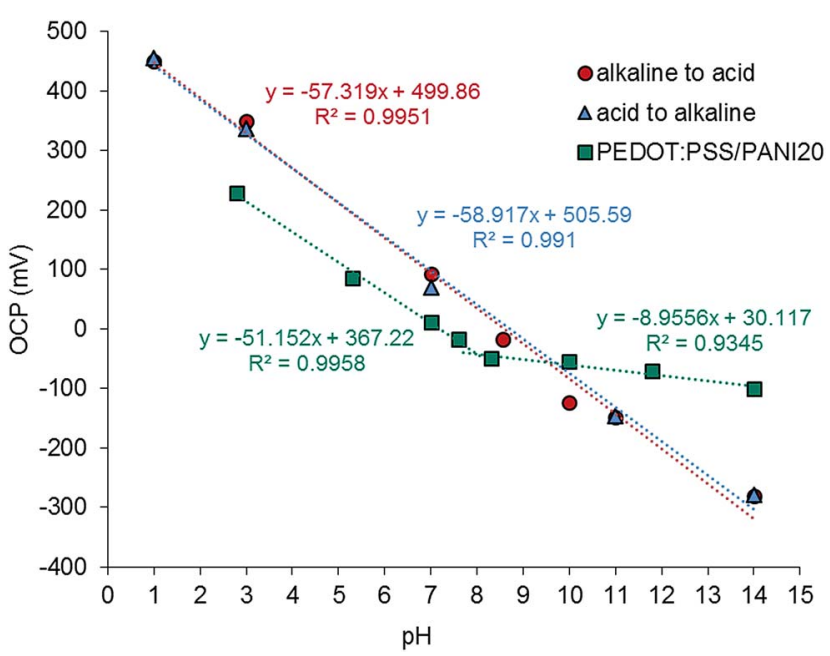

Fig. 7 Trends of open circuit potentials (OCP) versus $\mathrm{pH}$ for PEDOT:PSS/PANI electrode (measurements performed from alkaline to acidic or from acidic to alkaline) and PEDOT:PSS/PANI20 electrode (measurements performed from acidic to alkaline). plastic substrates, without metallic current collector. PEDOT layers have been obtained by ink-jet printing, and PANI was dipcoated. As can be seen in Fig. 8, PEDOT shows a linear trend of the potential equilibrium response as a function of $\mathrm{pH}$ with a slope of $10.5 \mathrm{mV} \mathrm{pH}^{-1}$ unit indicating a very low sensitivity toward $\mathrm{pH}$ variations, which can be attributed to the low conductivity of the ink-jet printed film. As highlighted in the literature, appreciable increasing in conductivity has been obtained only when PEDOT:PSS was modified with ethylene glycol, ${ }^{35}$ graphene oxide ${ }^{8}$ and dimethyl sulfoxide. ${ }^{1}$ As highlighted by different authors, the pristine PEDOT:PSS presents a coil structure were PEDOT grains are surrounded by a PSS insulating shield which prevents the interactions between the hydrophobic PEDOT and water. ${ }^{\mathbf{1 , 6 , 8 , 9 , 3 6}}$ Thus, the low $\mathrm{pH}$ responses may be attributed both to the low conductivity and to the inactivity of the $\mathrm{SO}_{3}{ }^{-}$groups of the PSS.

The dip-coated PANI shows a linear trend for $\mathrm{pH}$ between 2 and 7 with a slope of $50.7 \mathrm{mV} \mathrm{pH}^{-1}$ unit, while at higher values of $\mathrm{pH}$ the slope of OCP $v s$. $\mathrm{pH}$ decreases down to $7.7 \mathrm{mV} \mathrm{pH}^{-1}$ unit indicating a considerable loss of sensitivity at neutralalkaline pH. It is well known that PANI is a conducting polymer that shows a very high sensitivity toward $\mathrm{pH}$ : a linear dependence of OCP from $\mathrm{pH}$ in acidic solutions has been found for PANI electropolymerized on different substrates. ${ }^{37-41}$ At neutral or basic $\mathrm{pH}$ the sensitivity significantly decreased and, depending on the preparation conditions and on the substrates, the detection of $\mathrm{pH}$ is limited below $8-9$, while in the acidic range of $\mathrm{pH}$ PANI usually presents a near-Nerstian or a subNerstian behaviour. ${ }^{37-39,42}$ At higher values of $\mathrm{pH}$, the sensitivity decreases and a reduction of the slope in $\mathrm{pH} v s$. OPC curve has been often observed. In alkaline solution, the PANI becomes deprotonated and the conductivity of the films undergoes a dramatic decrease in magnitude.

Based on the above considerations, the high sensitivity of PEDOT:PSS/PANI in a wide range of $\mathrm{pH}$, can be really attributed to a synergistic effect. In the low $\mathrm{pH}$ range the response is due to protonation/deprotonation of amino groups of conducting

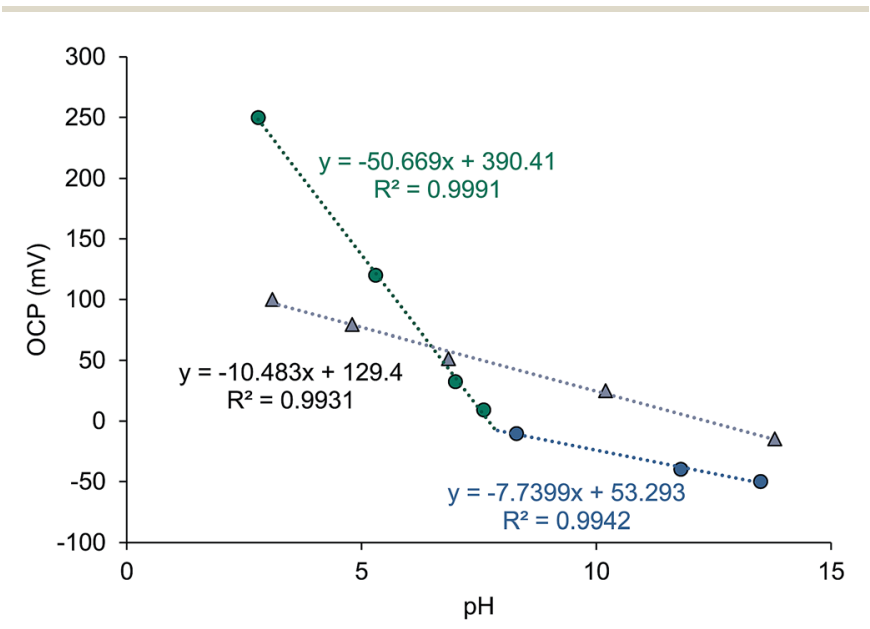

Fig. 8 Trends of open circuit potentials (OCP) versus $\mathrm{pH}$ for PEDOT:PSS (grey triangles) and PANI (green and blue circles) electrodes. 


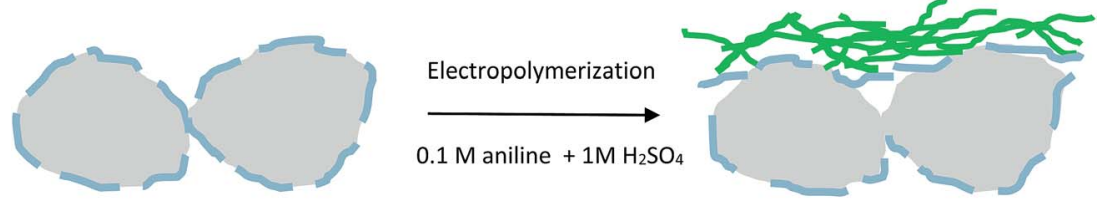

PEDOT $\simeq$ PSS $\rightleftharpoons$ PANI

Fig. 9 Sketch of the modifications of the conformation of pristine PEDOT:PSS after the electropolymerisation of PANI. The grey grain and the light blue lines stand for PEDOT and PSS respectively. The green fibers stand for PANI.

PANI. At neutral and alkaline $\mathrm{pH}$ the sensitivity of PEDOT/PANI can be explained considering that the ink-jet printed PEDOT:PSS contains large amounts of amorphous PSS as insulators which is added to dissolve PEDOT in water. When the PANI is electropolymerized on PEDOT:PSS, the positive charge in the PANI backbone may weaken the coulombic attraction between PEDOT and PSS creating conductive pathways which allows an increase of the conductivity of the pristine PEDOT:PSS structure (see scheme in Fig. 9), similarly to the results obtained with PEDOT:PSS treated with DMSO ${ }^{1}$ graphene oxide ${ }^{6,8}$ and ethylene glycol. ${ }^{9}$ Thus, when PANI in the organic bilayer is in its insulating form at alkaline $\mathrm{pH}$, the extended-coil conformation of PEDOT:PSS can guarantee higher conductivity while the negative charged groups $\mathrm{SO}_{3}{ }^{-}$can contribute to the responses at neutral and alkaline $\mathrm{pH}$ of the solution. It is worth observing that, when the PANI load increases, the behaviour of the bilayered film is similar to that of dip-coated PANI indicating that an excessive PANI load hinders the interactions between the negative charged groups $\mathrm{SO}_{3}{ }^{-}$and the solution.

\section{Conclusions}

Hybrid polymeric electrodes were obtained by inkjet printing of PEDOT:PSS combined with electropolymerisation of aniline. With this approach we obtained a bilayer electrode in which PEDOT:PSS was both active material and current collector, since metallic components were not used.

A thin film of PANI onto PEDOT:PSS was evidenced by SEM and Raman analyses. The electrodes were characterised by electrochemical impedance spectroscopy, which evidenced a large increase in the specific surface and a reduction of resistivity after coating of PEDOT:PSS substrates with PANI.

The electrodes were tested as $\mathrm{pH}$ sensors: the results show that the hybrid structures have linear response in $\mathrm{pH}$ window larger than those of either PEDOT:PSS or PANI electrodes, as well as higher sensitivity.

\section{Acknowledgements}

This work was partially funded by Regione Autonoma della Sardegna, Fundamental Research Programme, L. R. 7/2007 "Promotion of the scientific research and technological innovation in Sardinia'" under grant agreement CRP-59886 AMBROSIA Project and CRP-27388 BIOFET Project.

\section{References}

1 C. Deetuam, D. Weise, C. Samthong, P. Praserthdam, R. R. Baumann and A. Somwangthanaroj, J. Appl. Polym. Sci., 2015, 132, 42108-42117.

2 S. Rattan, P. Singhal and A. L. Verma, Polym. Eng. Sci., 2013, 53, 2045-2052.

3 M. Stavytska-Barba and A. M. Kelley, J. Phys. Chem. C, 2010, 114, 6822-6830.

4 T. Y. Wu, Z. Y. Kuo, J. J. Jow, C. W. Kuo, C. J. Tsai, P. R. Chen and H. R. Chen, Int. J. Electrochem. Sci., 2012, 7, 8076-8090.

5 Z. Xiong and C. Liu, Org. Electron., 2012, 13, 1532-1540.

6 X. Wu, J. Liu, D. Wu, Y. Zhao, X. Shi, J. Wang, S. Huang and G. He, J. Mater. Chem. C, 2014, 2, 4044-4050.

7 B. H. Zhang, G. P. Tan, Z. Y. Xie, W. Y. Wong, J. Q. Ding and L. X. Wang, Adv. Mater., 2012, 24, 1873-1877.

8 H. S. Dehsari, E. K. Shalamzari, J. N. Gavgani, F. A. Taromi and S. Ghanbary, RSC Adv., 2014, 4, 55067-55076.

9 J. Zhou, E. Q. Li, R. Li, X. Xu, I. Aguilar Ventura, A. Moussawi, D. H. Anjum, M. N. Hedhili, D. Smilgies, G. Lubineau and S. T. Thoroddsen, J. Mater. Chem. C, 2015, 3, 2528-2536.

10 Y. J. Xia, H. M. Zhang and J. Y. Ouyang, J. Mater. Chem., 2010, 20, 9740-9747.

11 W. Gaynor, G. F. Burkhard, M. D. McGehee and P. Peumans, Adv. Mater., 2011, 2, 2905-2910.

12 R. Jalili, J. M. Razal, P. C. Innis and G. G. Wallace, Adv. Funct. Mater., 2011, 21, 3363-3370.

13 H. Okuzaki, Y. Harashina and H. Yan, Eur. Polym. J., 2009, 45, 256-261.

14 T. Lindfors, S. Ervela and A. Ivaska, J. Electroanal. Chem., 2003, 560, 69-78.

15 R. Prakash, J. Appl. Polym. Sci., 2002, 83, 378-385.

16 H. C. Budnikov, G. A. Evtugyn and A. V. Porfireva, Talanta, 2012, 102, 137-155.

17 H. Shi, C. Liu, J. Xu, H. Song, B. Lu, F. Jiang, W. Zhou, G. Zhang and Q. Jiang, ACS Appl. Mater. Interfaces, 2013, 5, 12811-12819.

18 L. Zhang, H. Peng, P. A. Kilmartin, C. Soeller and J. TravasSejdic, Macromolecules, 2008, 41, 7671-7678.

19 H. Shi, C. Liu, J. Xu, H. Song, Q. Jiang, B. Lu, W. Zhou and F. Jiang, Int. J. Electrochem. Sci., 2014, 9, 7629-7643.

20 H. Shi, C. Liu, Q. Jiang, J. Xu, B. Lu, F. Jiang and Z. Zhu, Nanotechnology, 2015, 26, 245401-245409.

21 N. Plesu, A. Kellenberger, M. Mihali and N. Vaszilcsin, J. NonCryst. Solids, 2010, 356, 1081-1088. 
22 H. Lu, Y. Zhou, S. Vongehra, K. Hu and X. Meng, Synth. Met., 2011, 161, 1368-1376.

23 Q. Wang, Q. Yao, J. Chang and L. D. Chen, J. Mater. Chem., 2012, 22, 17612-17618.

24 Q. Zhao, R. Jamal, L. Zhang, M. Wang and T. Abdiryim, Nanoscale Res. Lett., 2014, 9, 557-566.

25 L. Fan, N. Zhang and K. Sun, Chem. Commun., 2014, 50, 6789-6792.

26 J. J. Shi, G. H. Yang and J. J. Zhu, J. Mater. Chem., 2011, 21, 7343-7349.

27 M. H. Pournaghi-Azar and B. Habibi, Electrochim. Acta, 2007, 52, 4222-4230.

28 H. K. Hassan, N. F. Atta and A. Galal, Int. J. Electrochem. Sci., 2012, 7, 11161-11181.

29 M. H. Yeh, C. P. Lee, C. Y. Chou, L. Y. Lin, H. Y. Wei, C. W. Chu, R. Vittal and K. C. Ho, Electrochim. Acta, 2011, 57, 277-284.

30 D. J. Yun, H. Ra and S. W. Rhee, Renewable Energy, 2013, 50, 692-700.

31 P. Danielsson, J. Bobacka and A. Ivaska, J. Solid State Electrochem., 2004, 8, 809-817.
32 J. R. Macdonald, Impedance spectroscopy - Emphasizing Solid Materials and Systems, Wiley-Interscience, New York, 1987.

33 J. Bobacka, A. Lewenstam and A. Ivaska, J. Electroanal. Chem., 2000, 489, 17-27.

$34 \mathrm{M}$. Asplund, H. von Holst and O. Inganas, Biointerphases, 2008, 3, 83-93.

35 J. Y. Ouyang, C. W. Chu, F. C. Chen, Q. Xu and Y. Yang, J. Macromol. Sci., Part A: Pure Appl.Chem., 2004, 41, 1497-1511.

36 Y. J. Xia and J. Y. Ouyang, ACS Appl. Mater. Interfaces, 2010, 2, 474-483.

37 T. Lindfors and A. Ivaska, J. Electroanal. Chem., 2002, 531, 43-52.

38 M. Kaempgen and S. Roth, J. Electroanal. Chem., 2006, 586, 72-76.

39 C. Ge, N. R. Armstrong and S. Scott Saavedra, Anal. Chem., 2007, 79, 1401-1410.

40 A. A. Karyakin, O. A. Bobrova, L. V. Luckachova and E. E. Karyakina, Sens. Actuators, B, 1996, 33, 34-38.

41 C. A. Lindino and L. O. S. Bulhoes, Anal. Chim. Acta, 1996, 334, 317-322.

42 B. Lakard, G. Herlem, S. Lakard, R. Guyetant and B. Fahys, Polymer, 2005, 46, 12233-12239. 